\title{
AVALIAÇÃO DA QUALIDADE DE VIDA DE PACIENTES COM INCOSTINÊNCIA URINÁRIA ATENDIDOS NO SETOR DE UROGINECOLOGIA DAS CLÍNICAS FAG
}

\section{LIFE QUALITY ASSESSMENT OF URINARY INCONTINENCE PATIENTS ATTENDED IN THE UROGYNECOLOGY SECTOR OF THE CLINICAL FAG}

\author{
Ivaneide Regina Luft', Lizyana Vieira²* \\ 1'Discente do curso de Fisioterapia do Centro Universitário Assis Gurgacz. ${ }^{2}$ Mestre,Docente do curso \\ de Fisioterapia do Centro Universitário Assis Gurgacz. \\ *Autor correspondente: lizyana@gmail.com
}

DOI: $10.35984 / f j h . v 2 i 1.115$

\section{RESUMO}

A incontinência urinária é uma condição constrangedora e tem consequências negativas na qualidade de vida, acarretando em quadros depressivos e isolamento de pessoas acometidas pela patologia. Dessa maneira o objetivo do estudo foi investigar o impacto da incontinência urinária na qualidade de vida dos pacientes atendidos no setor de Uroginecologia das Clinicas FAG de Cascavel-Paraná. Para isso, foi aplicado o questionário King's Health Questionnaire(KHQ), individualmente a dezesseis (16) pacientes, com predominância do gênero feminino 13 (81,25\%), com média de idade de 55,5 $\pm 13,7$ anos do setor de Uroginecologia das Clinicas FAG de Cascavel-Paraná. Conclui-se com esse estudo que a qualidade de vida é afetada negativamente principalmente nos quesitos que se referem ao desempenho de tarefas, sejam elas dentro ou fora de casa e na limitação física/social.

Palavras-chave: Pacientes; Incontinência urinária (IU), Qualidade de vida (QV).

\begin{abstract}
Urinary incontinence is an embarrassing condition and has negative consequences on quality of life, leading to depressive conditions and isolation of people affected by the disease. Thus, the objective of this study was to investigate the impact of urinary incontinence on the quality of life of patients treated at the Urogynecology sector of the FAG Clinics of Cascavel-Paraná. For this, the King's Health Questionnaire (KHQ) was applied individually to sixteen (16) patients, with a predominance of females 13 (81.25\%), with a mean age of $55.5 \pm 13.7$ years. of the Urogynecology sector of the FAG Clinics of Cascavel-Paraná. It is concluded with this study that the quality of life is negatively affected mainly in the questions that refer to the performance of tasks, whether inside or outside the home and in physical / social limitation.
\end{abstract}

Keywords: Patients; Urinary incontinence (UI), Quality of life (QOL).

\section{INTRODUÇÃO}

A Incontinência Urinária pode ser definida como "a perda de urina em quantidade e frequência suficientes para causar um problema social ou higiênico". 
Pode variar desde um escape ocasional até uma incapacidade total para segurar qualquer quantidade de urina. Ela se deve, com frequência, a alterações específicas do corpo em decorrência de doenças, uso de medicamentos ou pode representar o início de uma doença (MINISTÉRIO DA SAÚDE, 2006).

A incontinência urinária pode afetar qualquer pessoa, sendo as mulheres mais suscetíveis a apresentar essas condições, visto que apresentam uretra curta e fatores de riscos como assoalho pélvico submetido aos traumatismos obstétricos, lesões do nervo pudendo, das fáscias e músculos do assoalho pélvico, o avanço da idade, menopausa e alterações hormonais. A incontinência urinária apresenta-se não apenas como uma ameaça á auto-estima, mas também como fator de isolamento social e depressão (TAMANINI et al, 2003). Além disso, interfere negativamente, na qualidade de vida de muitas delas. O tratamento, que engloba técnicas fisioterapêuticas, medicamentosas ou cirúrgicas, possibilita as pessoas o retorno ás suas atividades, sem restrições, permitindo assim uma melhor qualidade de vida.

A incontinência urinária, com seus sintomas associados, pode causar significativo impacto na qualidade de vida e considerável variedade de percepções e respostas entre as pessoas (KLÜBER, 2004). Oliveira et al (2009), salienta a importância de se estudar a qualidade de vida pois a incontinência urinária é muitas vezes um motivo considerável e decisivo para internação em asilos ou casas de repouso.

A importância do levantamento de dados sobre o tema está em possibilitar aos portadores de incontinência urinaria uma melhor forma de tratar e, consequentemente uma melhor qualidade de vida.

Assim sendo, o objetivo do estudo foi investigar o impacto da incontinência urinária na qualidade de vida dos pacientes do setor de Uroginecologia das Clinicas FAG de Cascavel-Paraná.

\section{METODOLOGIA}

A pesquisa caracterizou-se como um estudo transversal, onde foram coletadas informações referentes a uma amostra dos pacientes do setor de Uroginecologia das Clinicas FAG no período de agosto a dezembro de 2018. A pesquisa foi aprovada pelo Comitê de Etica em Pesquisa em Seres Humanos - CEP mediante o número do CAAE: 96094118.2.0000.5219

Os pacientes foram entrevistados individualmente, em ambiente reservado, no setor de Uroginecologia das Clinicas FAG, em Cascavel, Paraná, antes ou após suas sessões de fisioterapia. Participaram da pesquisa pacientes com aptidão neurológica e psiquiátrica para responder o questionário, os mesmos aceitaram participar do presente estudo e, após a leitura e esclarecimento, concordaram e assinaram o Termo de Consentimento Livre e Esclarecido (TCLE).

A pesquisa foi realizada através de entrevista face-a-face, utilizando o questionário King's Health Questionnaire (KHQ) validado por Tamanini et al. (2003)(Anexo 2);O KHQ é composto por nove domínios que relatam, respectivamente, a percepção da saúde, o impacto da incontinência, as limitações do desempenho das tarefas, a limitação física, a limitação social, o relacionamento pessoal, as emoções, o sono e a energia e as medidas de gravidade. A todas as respostas foram atribuídos valores numéricos, somados e avaliados por domínio. Os valores foram, então, calculados por meio de fórmula matemática, obtendo-se assim, o escore de qualidade 
de vida, que varia de 0 a 100, considerando-se que quanto maior o número obtido, pior a qualidade de vida.

\section{RESULTADOS E DISCUSSÃO}

Participaram do estudo 16 indivíduos, com predominância do gênero feminino $13(81,25 \%)$, com média de idade de 55,5 $\pm 13,7$ anos. A expectativa de vida é um processo que vem aumentando com o decorrer do tempo, no Brasil é acompanhado por modificações no perfil de saúde de sua população e predomínio de doenças crônicas, com limitações funcionais, incapacidades e maiores gastos e desafios para o sistema de saúde. À medida que o ser humano envelhece, quer continuar tendo força, resistência, flexibilidade e mobilidade para permanecer ativo e independente de modo a poder atender as próprias necessidades pessoais e domésticas, como fazer uma simples compra ou participar de atividades recreativas e esportivas (RIKLI; JONES, 2008).

Quando perguntado, "como avaliaria sua saúde hoje?", $50 \%$ (8) relataram como sendo "normal" seu estado de saúde naquele momento, e 25\% (4) classificaram como "Ruim". Quanto à interferência da IU em suas vidas, 68,8\% (11) relataram haver "muita" interferência e, apenas um (6,3\%) paciente referiu que a incontinência não afetava a sua vida.

Para o domínio "Desempenho das tarefas", os participantes tinham que responder a dois subitens: Quanto seu problema de bexiga atrapalha suas atividades em casa e quanto seu problema de bexiga atrapalha suas tarefas fora de sua residência. Respectivamente, 31,3\% (5) relataram haver "um pouco" de interferência nas atividades em casa e, 50\% (8) relataram "muita" interferência em suas tarefas fora de sua residência. Rufino Netto (1994) denomina qualidade de vida (QV, de ora em diante) boa ou excelente aquela que ofereça um mínimo de condições para que os indivíduos nela inseridos possam desenvolver o máximo de suas potencialidades. Sendo assim, indivíduos que tem seu desempenho em tarefas dentro ou fora de suas residências afetadas, tem a sua qualidade de vida diminuída.

Sobre limitação física, se "seu problema de bexiga atrapalha suas atividades físicas como: fazer caminhada, correr, fazer algum esporte, etc", $37,5 \%$ (6) responderam atrapalhar "Muito"; e quando perguntado se "seu problema de bexiga atrapalha quando você quer fazer uma viagem", $50 \%$ (8) responderam atrapalhar "Muito". Cordeiro (2008), em seu estudo, observou que 77 a $87 \%$ das mulheres entrevistadas relataram sofrer interferências na limitação física, seja em atividades dentro ou fora das suas residências.

Sobre limitação social, quando perguntado se "seu problema de bexiga atrapalha quando você vai a igreja, reunião, festa", 43,8\% (4) responderam "Muito", e quando perguntado "você deixa de visitar seus amigos por causa do problema de bexiga", 62,5\% (10) responderam "Não" deixar de visitar. Segundo Lopes e Higa (2006), muitos portadores da IU acabam deixando de ter uma vida social como visitar a amigos, realizar viagens e até dormir fora de casa por causa do impacto que a disfunção ocasiona. No mesmo estudo foi observado que 15 a $30 \%$ das mulheres incontinentes apresentam alterações no âmbito profissional, doméstico, físico e têm disfunção sexual ocasionada pela IU. Já no estudo de Barentsen et al. (2012), observou-se que 25 a 50\% das incontinentes idosas referiam sentir vergonha por ter a disfunção e por causa deste sentimento passaram a se isolar socialmente. 
No aspecto geral, a incontinência apresenta várias limitações como, por exemplo, prática de esportes, tarefa doméstica, estado emocional e vida sexual. $O$ desconforto social e higiênico se dá principalmente por medo da perda e do cheiro da urina e a utilização de protetores (absorventes) e frequentes trocas de roupa (LOPES, 2006).

O âmbito "relações pessoais" é dividido em três subitens, onde foi perguntado "o quanto o problema de bexiga atrapalha na vida sexual" e $37,5 \%$ (6) dos entrevistados relataram "atrapalhar muito" a vida sexual; Para lidar com a incontinência urinária no dia-a-dia muitas mulheres desenvolvem estratégias, seja evitar a relação sexual ou até diminuir a frequência, reduzir a ingestão de líquidos, não frequentar locais sem banheiros e muitas chegam a não relatar o seu problema para os próprios parceiros (BEJl et al,.2005). No segundo subitem foi perguntado aos indivíduos se "o problema de bexiga atrapalha na vida com seu companheiro", 56,3\% (9) das pessoas afirmaram "não atrapalhar", e no terceiro subitem foi perguntado "se o problema de bexiga incomoda seus familiares", sendo $87,5 \%$ (14) das respostas "não incomoda". LOPES (2006), afirma que sintomas irritativos como noctúria e urgência muitas vezes atrapalham relacionamentos sociais e o sono, podendo assim evoluir para um isolamento social e depressão.

Quanto aos problemas de bexiga e o quanto eles afetam os indivíduos entrevistados, sobre a frequência: Você vai muitas vezes ao banheiro? $62,5 \%$ (10) responderam "Muito", e apenas 6,3\% (1) respondeu "Não" ir muitas vezes ao banheiro; Noctúria: você levanta a noite para urinar? 43,8\% (7) responderam "Muito"; o que teria uma interferência negativa na qualidade do sono do indivíduo; e quanto a Urgência: você tem vontade forte de urinar e muito difícil de controlar? 62,5\% (10) responderam "Muito"; Bexiga hiperativa: você perde urina quando você tem muita vontade de urinar? 56,3\% (9) responderam "Muito"; No estudo de Honório e Santos (2009), foi encontrado que as principais queixas dos incontinentes referentes a não sair de casa com frequência era a necessidade constante de procurar um banheiro e o receio de não conseguir encontrar ou não dar tempo por causa da forte vontade de urinar.

Quanto à incontinência urinária de esforço: você perde urina com atividades físicas como: tossir, espirrar, correr? 50\% (8) responderam "Muito"; Enurese noturna: você molha a cama à noite? 62,5\% (10) responderam "Não"; Incontinência no intercurso sexual: você perde urina durante a relação sexual? 56,3\% (9) responderam "Não"; Infecções frequentes: você tem muitas infecções urinárias? $37,5 \%$ (6) responderam "Não"; Dor na bexiga: você tem dor na bexiga? 31,3\% (5) responderam "Não"; E por fim, outros: você tem algum outro problema relacionado a sua bexiga? 93,8\% (15) responderam "Não" ter outro problema relacionado.

Relacionado á emoções, os entrevistados responderam as seguintes perguntas: "você fica deprimida (o) com seu problema de bexiga?", 31,3\% (5) responderam "Não" ficar deprimidos e 25\% (4) responderam que ficam "muito" deprimidos com o problema urinário; "Você fica ansiosa (o) ou nervosa (o) com seu problema de bexiga?", e 31,3\% (2) responderam "um pouco"; Também foi perguntado "Você fica mal com você mesma (o) por causa do seu problema de bexiga?", 37,5\% disseram "Não" e 31,3\% (5) disseram "às vezes" ficar mal por conta do problema. Abreu et al. (2007) relatam sobre o perigo das incontinentes aumentarem a tendência ao isolamento quando passam a sofrer com a disfunção.

No estudo de Rett et al. (2007), eles descrevem que a incontinência urinária é tão prejudicial que acaba afetando suas portadoras não apenas na parte física, mas também no âmbito da sua saúde mental, seja pela depressão ou pela perda da autoestima, em maior ou menor proporção. 
No domínio "sono/energia" foi perguntado aos participantes se "o seu problema de bexiga atrapalha no sono", 62,5\% (10) responderam que "não" atrapalha. Também foi perguntado se sentiam-se desgastados ou cansados, e $50 \%$ (8) dos indivíduos responderam que "não". Os resultados encontrados divergem do estudo de Oliveira et al. (2009), que encontrou um impacto de 52,94\%, talvez pelo fato de o alto índice de portadores referirem noctúria, que compromete seu sono, deixando-os desgastados e cansados, o que pode interferir na qualidade de vida.

Por último, foram abordadas algumas situações que poderiam acontecer com os indivíduos e o quanto elas ocorreriam; Quanto ao uso de fralda, forro, absorvente tipo Moldes para manter-se seco, 43,8\% (7) dos entrevistados admitiram fazer o uso de algum tipo de proteção "sempre"; Com relação ao controle da quantidade de líquido ingerido, 56,3\% (9) disseram não controlar a quantia de líquido que bebe; Quanto à necessidade de trocar a roupa íntima quando ficam molhados, houve uma igualdade nas respostas "às vezes" e "sempre" com 37,5\% (6) dos indivíduos tendo a necessidade de fazer a troca de roupa íntima. E quanto à preocupação de estar cheirando urina, 68,8\% (11) dos pacientes revelaram "sempre" estarem preocupados com o cheiro de urina que possam estar exalando

Leung (1997) afirma que, apesar de a IU ser comum, 50 a $70 \%$ de pessoas com esta queixa não procuram ajuda, por acreditarem ser uma alteração normal decorrente do envelhecimento, ao passo que outras se sentem constrangidas em revelá-la aos profissionais de saúde.

Nesse sentido, é importante salientar que a IU não é uma consequência normal do envelhecimento, mas de uma doença que pode ser curada, utilizando-se de técnicas fisioterapêuticas, medicamentosas ou cirúrgicas.

\section{CONSIDERAÇÕES FINAIS}

Conclui-se com esse estudo que a qualidade de vida é afetada negativamente principalmente nos quesitos que se referem ao desempenho de tarefas, sejam elas dentro ou fora de casa e na limitação física/social.

\section{REFERÊNCIAS}

ABREU, N.S et al. Qualidade de vida na perspectiva de idosos com incontinência urinária.Rev. bras. Fisioter., 11(6). 2007.

BEJI, N.K; YALCIN, O; ERKAN, H.Á; KAYIR, A. Effect of urinary leakage on sexual function during sexual intercourse. Uro Int, 74(3):250-5. 2005

BEZERRA, Maria Rita Lima et al. Qualidade de vida: um instrumento para promoção de saúde. Revista Baiana de Saúde Pública, v.32, n.2, p.232-240 maio/ago. 2008.

BRUNA, Maria Helena Varella. Incontinência Urinária. Disponível em:

< https://drauziovarella.uol.com.br/doencas-e-sintomas/incontinencia-urinaria/>. Revisado. 06 de março de 2018. Acesso em: 01 jun 2018.

CORDEIRO, G. - Qualidade de Vida na Perspectiva de Mulheres Climatéricas com Incontinência Urinária. Dissertação (Programa de Pós-Graduação em Saúde 
Materno-Infantil). Universidade Federal do Maranhão, São Luís - MA, 2008. https://tedebc.ufma.br/jspui/handle/tede/tede/1119

COSTA, Mayara Leal Almeida; Qualidade de vida na terceira idade: a psicomotricidade como estratégia de educação em saúde. Dissertação apresentada para Mestrado. Lisboa: 2011. http://hdl.handle.net/10437/2230

FONSECA, Eliana Suelotto Machado et al. Validação do questionário de qualidade de vida (King's Health Questionnaire) em mulheres brasileiras com incontinência urinária. RevBrasGinecol Obstet. 27(5): 235-42: 2005. http://dx.doi.org/10.1590/S010072032005000500002

GIRÃO, Manoel João Batista Castello. DE LIMA, Geraldo Rodrigues. BARACAT, Edmund Chada.Ginecologia. 1르. Ed. Barueri, Manole: 2009.

HONÓRIO, M. O.; SANTOS, S. M. A. Incontinência urinária e envelhecimento: impacto no cotidiano e na qualidade de vida. Rev Bras Enferm, v. 62, n. 1, p. 51-56, 2009. http://dx.doi.org/10.1590/S0034-71672009000100008

KLÜBER, L. A influência da Fisioterapia na qualidade de vida de mulheres com incontinência urinária: revisão. Revista do Crefito 5, Curitiba, v. 2, n. 8, dez. 2004.

LEUNG, K. S. et al. Incontinência urinária: um problema ignorado em pacientes idosos. HKMJ, Honk Kong, v. 3: 1997.

LOPES, Maria Helena Baena de Moraes; HIGA, Rosângela. Restrições causadas pela incontinência urinária à vida da mulher. Revista da Escola de Enfermagem da USP, v.40, n.1, p.34-41. São Paulo: 2006. http://dx.doi.org/10.1590/S008062342006000100005

MINAYO, Maria Cecília; Qualidade de vida e saúde: um debate necessário. Ciência \& Saúde Coletiva. Disponível em: $<$ http://www.faculdadeatlantico.com.br/site antigo/downloads/Qualidadedevidaesaud e.pdf>. Acesso em: 01 jun 2018. http://dx.doi.org/10.1590/S141381232000000100002 .

Ministério da Saúde. Envelhecimento e Saúde da Pessoa Idosa. Brasília-DF: 2006. Disponível

em:<http://bvsms.saude.gov.br/bvs/publicacoes/evelhecimento saude pessoa idos a.pdf $>$ Acesso em 06 jun 2018.

OLIVEIRA, Sheila Gemelli; Avaliação da Qualidade de Vida de Poortadores de Incontinência Urinária. RBCEH, v. 6, n. 1, p. 34-41. Passo Fundo, 2009. https://doi.org/10.5335/rbceh.2012.251.

PALMA, Paulo Cesar Rodrigues. Urofisioterapia Aplicações clínicas das técnicas fisioterapêuticas nas disfunções miccionais e do assoalho pélvico. $2^{a}$ Ed São Paulo: 2014. 
POLDEN, Margaret; MANTLE, Jill. Fisioterapia em obstetrícia e Ginecologia. São Paulo, Santos: 2002.

RETT, M.T; et al. Qualidade de Vida em Mulheres após Tratamento da Incontinência Urinária de Esforço com fisioterapia.RBGO Revista Brasileira de Ginecologia e Obstetrícia, v.29, p. 134-140, 2007. http://dx.doi.org/10.1590/S010072032007000300004

RIBEIRO, R|icardo Muniz. Uroginecologia e cirurgia vaginal. São Paulo, Roca: 2001.

RIKLI; R. E. JONES, C. J. Teste de Aptidão Física para Idosos. Cap 05, pág 100 182. São Paulo: Manole, 2008.

RUFINO NETO, A. Qualidade de Vida: Compromisso Histórico da Epidemiologia. Belo Horizonte: Coopmed/Abrasco, 1994, Cap.1, p.11-18. http://dx.doi.org/10.1590/S0102-311X1995000100026.

TAMANINI, J. T. N. et al. Validação do "king's Health Questionnaire“ para o português em mulheres com incontinência urinária. Ver. Saúde Pública [online]. V.37,n.2,abr 2003.

Disponível

em:<http://www.scielo.br/scielo.php?script=sci_arttext\&pid=S0034-

$89102003000200007 \&$ Ing=en\&nrm=iso $>$. Acesso em 26 de maio de 2018. http://dx.doi.org/10.1590/S0034-89102003000200007. 